\title{
EL PAPEL de LA AUTOESTIMA EN LA PREVENCIÓN DEL VIH DE JÓVENES ESPAÑOLES
}

\author{
Ruiz-Palomino, Estefanía \\ Departamento de Psicología Básica, Clínica y Psicobiología, Universitat Jaume I de Castelló (UJI) \\ Ballester-Arnal, Rafael \\ Departamento de Psicología Básica, Clínica y Psicobiología, Universitat Jaume I de Castelló (UJI) \\ Gil-Llario, María Dolores \\ Departamento de Psicología Departamento de Psicología Evolutiva y de la Educación, \\ Universitat de València (UV) \\ Giménez-García, Cristina \\ Departamento de Psicología Básica, Clínica y Psicobiología, Universitat Jaume I de Castelló (UJI) \\ ruiz@uji.es, salusex@uji.es
}

https://doi.org/10.17060/ijodaep.2017.n1.v2.914

Fecha de Recepción: 11 Enero 2017

Fecha de Admisión: 1 Abril 2017

\section{RESUMEN}

La autoestima influye en el desarrollo de la sexualidad y de las relaciones interpersonales de los jóvenes. Con el objetivo de examinar los efectos de la autoestima en la prevención del VIH, se evaluaron a 467 jóvenes de la Comunidad Valenciana $\left(M_{\text {edad }}=20,62 ; \mathrm{DT}=2,14\right)$. Se utilizó la Escala de Autoestima de Rosenberg y el Cuestionario de Prevención del Sida de Ballester et al. (2007). Los resultados muestran relaciones positivas estadísticamente significativas de la autoestima con autoeficacia en el uso del preservativo $(p=.000)$, competencia percibida $(p=.041) 0$ número de parejas sexuales $(p=.001)$. Por el contrario, se encuentran relaciones negativas estadísticamente significativas con solidaridad hacia las personas que viven con VIH ( $p=.013)$, barreras percibidas para utilizar el preservativo $(p=.030)$, intención de usar el preservativo con parejas esporádicas $(p=.043)$ y uso del preservativo con pareja estable $(p=.021)$. Resulta necesario atender a la autoestima para diseñar intervenciones psicológicas eficaces para la prevención del VIH.

Palabras clave: autoestima, jóvenes, prevención VIH, intervenciones psicológicas.

\section{ABSTRACT}

The role of self-esteem in HIV prevention among Spanish young people

Self-esteem influences on sexual development and relationships of young people. Aims to examine the effects of the self-esteem on HIV prevention, 467 young people from the Valencian Community $\left(\mathrm{M}_{\text {age }}=20.62\right.$; $\left.\mathrm{SD}=2.14\right)$ completed the Rosenberg Self-esteem Scale and the Aids 


\section{EL PAPEL de LA AUtOESTIMA EN LA PREVENCIÓN DEL VIH DE JÓVENES ESPAÑOLES}

Prevention Questionnaire by Ballester et al. (2007). Results showed statistically significant positive correlation between self-esteem and self-efficacy in condom use $(p=.000)$, perceived competence $(p=.041)$ or number of sexual partners $(p=.001)$. On the other hand, statistically significant negative correlation were found between solidarity towards people living with HIV $(p=.013)$, perceived barriers to condom use $(p=.030)$, behavioral intention condom use with sporadic partners $(p=$ .043 ) and condom use with steady partner $(p=.021)$. It is important to address self-esteem to design effective psychological interventions for HIV prevention.

Keywords: Self-esteem, youth, HIV prevention, psychological interventions.

\section{ANTECEDENTES}

Tradicionalmente se ha considerado a la autoestima como un componente clave de la salud y el bienestar psicológico (Jahoda, 1958). Este constructo ha sido el elemento principal de algunos programas de promoción de la salud en jóvenes relacionados con la prevención de la violencia, el consumo de sustancias y el comportamiento sexual de riesgo (0xley, 2001). Con una orientación humanista y unidimensional, Rosenberg (1989) señaló la necesidad de definir la autoestima desde la perspectiva del valor, es decir, como los sentimientos de valía personal y respeto hacia uno mismo. Estos valores servirán de principios, normas y criterios para la construcción del propio self. Así pues, una alta autoestima se expresa mediante la auto-aceptación y la responsabilidad de las propias acciones, la auto-motivación, la capacidad de tolerar el estrés y de controlar las circunstancias. Por el contrario, una baja autoestima puede llevar a sentimientos de apatía, aislamiento y pasividad, falta de confianza, locus de control externo y ambigüedad sobre el propio valor personal. No obstante, sigue siendo un constructo psicológico complejo de definir y evaluar (Mruk, 2013). Como indican Ortega, Mínguez y Rodes (2000), su conceptualización ha variado desde un enfoque actitudinal hasta otros que la entendían como una función de la personalidad. De un modo u otro, el aspecto que prevalece en todas las definiciones es el origen social de la misma.

La literatura científica en el ámbito de la autoestima y la conducta sexual ha reflejado resultados en tres direcciones diferentes. Una parte de los estudios sugiere que una baja autoestima se asocia con relaciones sexuales precoces, mayor número de parejas sexuales, dificultades para la asertividad sexual y mayor frecuencia de prácticas sexuales de riesgo (Enejoh, Pharr, Mavegam, Olutola, Karick y Ezeanolue, 2016; Ethier, Kershaw, Lewis, Milan, Niccolai y Ickovics, 2006; Klein, Elifson y Sterk, 2010; Ramiro, Teva, Bermúdez y Buela-Casal, 2013; Sylvester, 2014). Otra parte de los estudios demuestran que tener una alta autoestima puede influir negativa o positivamente en la salud sexual. Por ejemplo, Salazar, Crosby, DiClemente, Wingood, Lescano, Brown et al. (2005), Villegas, Cianelli, Gonzalez-Guarda, Kaelber, Ferrer y Peragallo (2013) o Yang, Dedovic y Zhang (2010), mostraron cómo mayores puntuaciones en autoestima se relacionaban con actitudes positivas hacia el preservativo y mayor percepción de eficacia en la negociación de su uso. Sin embargo, Ugoji (2014) observa que los jóvenes con alta autoestima pueden realizar conductas sexuales de riesgo por su baja percepción de vulnerabilidad al riesgo. La tercera corriente de estudios muestra que no hay asociación entre la autoestima y las conductas sexuales de riesgo (Træen, Hald, Noor, lantaffi, Grey, y Rosser, 2014), o que estas asociaciones no son directas (MacDonald y Martineau, 2002).

\section{OBJETIVOS}

Por todo lo anterior, el objetivo de este estudio es examinar el rol que juega la autoestima en algunos de los factores cognitivos, afectivos y conductuales principales de la prevención del VIH en jóvenes. 


\section{PARTICIPANTES}

En el presente estudio participaron 467 jóvenes universitarios entre 17 y 26 años $\left(M_{e d a d}=20,62\right.$; $\left.D T=2,14\right)$ de la Comunidad Valenciana que participaban o habían participado en actividades organizadas por SaluSex-Unisexsida (Unidad de Investigación en Salud y Sexualidad de la Universitat Jaume I de Castellón). Del total de la muestra, 274 eran mujeres (58.7\%) y 193 hombres (41.3\%). La mayor parte de la muestra se identificaba con una orientación heterosexual $(91,2 \%)$, seguida de homosexual $(5,6 \%)$ y bisexual $(3,2 \%)$. No obstante, un $11,8 \%$ indicó que había mantenido relaciones sexuales con personas de su mismo sexo.

\section{METODOLOGÍA \\ Instrumentos de evaluación}

Escala de Autoestima de Rosenberg (1965) (versión de Martín-Albo, Núñez, Navarro y Grijalvo, 2007). Cuestionario compuesto de 10 ítems con enfoque unidimensional y elaborado desde una concepción fenomenológica de la autoestima que se centra en los sentimientos de respeto y aceptación de la persona hacia sí misma. Dispone de un formato de respuesta tipo Likert de 4 puntos, donde 1 significa muy de acuerdo y 4 significa totalmente en desacuerdo. Para su corrección, deben invertirse las puntuaciones de los ítems enunciados negativamente $(2,5,8,9,10)$ y posteriormente sumarse todos los ítems. La puntuación total, por tanto, oscila entre 10 y 40 puntos.

Cuestionario de Prevención del Sida de Ballester, Gil y Giménez (2007). Instrumento multidimensional compuesto de 65 ítems que integran métodos de evaluación cuantitativos y cualitativos. Se evalúan los principales componentes relacionados con la prevención del sida que aparecen en los diferentes modelos de la conducta de salud. Para el presente estudio se han utilizado:

Componentes cognitivos. Creencia de autoeficacia en el uso del preservativo, competencia percibida, información y conocimientos sobre el VIH, vulnerabilidad y gravedad percibida a la infección por VIH, empatía hacia las personas que viven con VIH.

Componentes afectivos-motivacionales. Percepción de ventajas e inconvenientes hacia el uso del preservativo, temor percibido a la infección por VIH, intención o motivación de conducta de uso del preservativo.

Componentes conductuales. Número de parejas y prácticas sexuales, uso del preservativo en diferentes prácticas sexuales, asertividad sexual con la pareja.

\section{Procedimiento}

Los datos utilizados en el presente estudio corresponden a la fase pre-test de un proyecto de investigación más amplio, que tenía como objetivo final la evaluación de la eficacia a corto, medio y largo plazo de una intervención preventiva breve dirigida a población joven. La obtención de los datos se realizó en papel y estuvo supervisada por un psicólogo de la salud entrenado. Los instrumentos utilizados en el estudio estaban integrados en una batería de cuestionarios, cuya cumplimentación tuvo una duración media de 90 minutos. Los participantes firmaron el consentimiento informado y se siguieron las directrices de la Ley Orgánica de Protección de Datos (LOPD).

\section{RESULTADOS}

Tras el análisis de correlaciones entre la autoestima y los distintos factores cognitivos se observan relaciones estadísticamente significativas con signo positivo con la puntuación total de creencia de autoeficacia en el uso del preservativo $(r=0.224 ; p=.000)$ y la competencia percibida para el uso del preservativo ( $r=0.096 ; p=.041)$. En concreto, aparece una relación significativa a nivel estadístico con casi todas las habilidades evaluadas relacionadas con el uso del preservativo en distintos momentos de una relación sexual (ver Tabla 1). Por el contrario, se encuentran relaciones nega- 


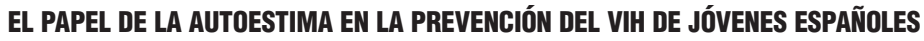

tivas estadísticamente significativas con el sentimiento de solidaridad hacia las personas que viven con VIH ( $r=-0.117 ; p=.013)$. No existen relaciones estadísticamente significativas con información sobre VIH ( $r=-0.041 ; p=.385)$, vulnerabilidad percibida a la infección por VIH $(r=-0.045 ; p=.333)$, gravedad atribuida a la enfermedad del sida ( $R h 0=0.034 ; p=.463$ ) y empatía hacia las personas que viven con VIH $(r=-0.001 ; p=.990)$.

Tabla 1.

Correlación entre autoestima y facetas de la autoeficacia en el uso del preservativo

\begin{tabular}{lc}
\hline & $\begin{array}{c}\text { Autoestima } \\
\boldsymbol{r}(\boldsymbol{p})\end{array}$ \\
\hline $\begin{array}{l}\text { 1. Me siento o sentiría cómodo o seguro a la hora de comprar } \\
\text { preservativos }\end{array}$ & $\mathbf{0 . 1 5 0}(. \mathbf{0 0 1})$ \\
$\begin{array}{l}\text { 2. Me resultaría cómodo hablar acerca de la utilización de } \\
\text { preservativo con una pareja antes de comenzar una relación sexual }\end{array}$ & $0.034(.472)$ \\
$\begin{array}{l}\text { 3. Si tengo que sugerir a una pareja que usemos el preservativo, } \\
\text { tengo o tendría miedo de que me rechazase* }\end{array}$ & $\mathbf{0 . 1 0 9 ( . 0 2 0 )}$ \\
$\begin{array}{l}\text { 4. No me siento o sentiría seguro a la hora de sugerir el } \\
\text { preservativo a una nueva pareja, ya que podría pensar que lo hago } \\
\text { porque creo que tiene una ITS* }\end{array}$ & $\mathbf{0 . 1 0 5 ( . 0 2 4 )}$ \\
$\begin{array}{l}\text { 5. Estoy seguro de que recordaría usar el preservativo aunque } \\
\text { haya consumido alcohol u otras drogas } \\
\text { 6. Me siento o sentiría incómodo a la hora de ponerme el condón o } \\
\text { ponérselo a mi pareja* }\end{array}$ & $\mathbf{0 . 1 1 6 ( . 0 1 3 )}$ \\
$\begin{array}{l}\text { 7. Estoy seguro de que podría parar aún en el momento de mayor } \\
\text { excitación para ponerme el condón o ponérselo a mi pareja }\end{array}$ & $\mathbf{0 . 1 3 3 ( . 0 0 4 )}$ \\
\hline * İtems invertidos. &
\end{tabular}

En relación con los factores afectivo-motivacionales, se obtienen relaciones negativas estadísticamente significativas con las barreras percibidas para utilizar el preservativo $(r=-0.102 ; p=.030)$, el uso del preservativo con pareja estable ( $\mathrm{Rho}=-0.114 ; \mathrm{p}=.021)$ y la intención de usar el preservativo con parejas esporádicas (Rho=-0.147; $p=.043$ ). No se observan relaciones significativas a nivel estadístico con la intención de conducta ni con la conducta manifiesta en el resto de situaciones sexuales evaluadas (ver Tabla 2), ventajas atribuidas al uso del preservativo $(r=-0.051 ; p=.275)$ y vulnerabilidad percibida a la infección por VIH ( $r=-0.045 ; p=.333)$.

Tabla 2.

Correlación entre autoestima, intención de conducta y conducta del preservativo en distintas situaciones sexuales

\begin{tabular}{|c|c|c|}
\hline & \multicolumn{2}{|c|}{ Autoestima } \\
\hline & $\begin{array}{l}\text { Conducta } \\
\text { Rho (p) }\end{array}$ & $\begin{array}{c}\text { Intención de } \\
\text { conducta } \\
\text { Rho }(p)\end{array}$ \\
\hline Coito vaginal & $-0.035(.480)$ & $0.086(.260)$ \\
\hline Sexo oral & $0.005(.920)$ & $-0.043(.570)$ \\
\hline Coito anal & $0.018(.820)$ & $-0.036(.579)$ \\
\hline Relaciones sexuales con pareja estable & $-0.114(.021)$ & $0.009(.905)$ \\
\hline Relaciones sexuales con pareja esporádica & $0.073(.181)$ & $-0.147(.043)$ \\
\hline $\begin{array}{l}\text { Relaciones sexuales bajo los efectos del alcohol u } \\
\text { otras drogas }\end{array}$ & $0.063(.229)$ & $-0.060(.423)$ \\
\hline
\end{tabular}


Por último, con respecto a los factores conductuales, existen relaciones estadísticamente significativas con signo positivo con número de parejas sexuales $(r=0.152 ; p=.001)$, con la realización de prácticas sexuales como la masturbación ( $R h 0=0.122 ; p=.009) 0$ el sexo oral ( $R h 0=0.098 ; p=.036$ ), y con la asertividad sexual a la hora de preguntar a las parejas sobre el VIH y las pruebas de detección de anticuerpos (Rho=0.128; $\mathrm{p}=.007$ ). Por el contrario, aparecen relaciones estadísticamente significativas con signo negativo con el uso del preservativo con pareja estable (Rho=-0.114; $\mathrm{p}=$.021) (ver Tabla 2).

\section{DISCUSIÓN}

La autoestima se construye durante la adolescencia a partir de la valoración del propio comportamiento y de la interiorización de la opinión de los demás. Este constructo ha sido clásicamente considerado como un factor protector o de amortiguación de las experiencias de riesgo, como así lo demuestran Orth, Robins y Widaman (2012) con colectivos jóvenes de riesgo. Sin embargo, la profunda investigación sobre su conceptualización ha dado lugar a diversas facetas características de la autoestima, que le otorgan diferentes roles en el ámbito de la conducta sexual de riesgo (Ortega et al., 2000).

En general, nuestros resultados demuestran que la influencia positiva o negativa de la autoestima varía en función de la variable en la que se exprese. Por una parte, se observa que los jóvenes con una autoestima más elevada se sienten más competentes sexualmente para usar el preservativo en los diferentes momentos de la relación sexual (por ejemplo, comprar, negociar, etc.) o para hablar sobre el VIH. Este hecho ha sido demostrado previamente con jóvenes en algunos estudios como los de Crosby, Yarber, Sanders y Graham (2005) o Villegas et al. (2013), cuya autoestima ha favorecido la toma de decisiones sexuales saludables y la resistencia a presiones externas. Por otra parte, se obtiene que los jóvenes con mayor autoestima demuestran menor empatía y solidaridad hacia las personas que viven con VIH. Quizá este aspecto pueda ser explicado por la relación entre alta autoestima y tendencia a la auto-disciplina, control y responsabilidad sobre los propios actos que sugiere Mruk (2013), es decir, puede que los jóvenes con mayor autoestima sean menos flexibles cognitivamente, sientan rechazo y culpabilicen a las personas que han realizado conductas sexuales de riesgo. Por último, los jóvenes con mayor autoestima confirman haber tenido más frecuencia de parejas sexuales, contrariamente a lo obtenido por autores como Enejoh et al. (2016). Y también se encuentra una tendencia a realizar más prácticas sexuales de riesgo con parejas estables y a tener menos intención de realizar conductas sexuales seguras con parejas esporádicas, algo contrario también a lo encontrado por Klein et al. (2010), Ramiro et al. (2013) o Sylvester et al. (2014). Puede que características de las personas con alta autoestima, como la cordialidad, la extraversión, la sociabilidad o las emociones positivas, puedan explicar la relación encontrada en la muestra de jóvenes evaluados. 0 también que niveles altos de autoestima lleven a subestimar el riesgo de infección. E incluso que una elevada autoestima, en el caso de la persona que no quiere utilizar el preservativo, le lleve a una mayor capacidad para imponer su deseo a la pareja.

\section{CONCLUSIONES}

Dada la importancia que la opinión acerca de uno mismo tiene en el desarrollo de la sexualidad y de las relaciones interpersonales de los jóvenes, se considera necesario incluir este componente en el diseño de las intervenciones psicológicas, teniendo en cuenta la variabilidad de su influencia en los diferentes factores relacionados con la prevención del VIH.

\section{AGRADECIMIENTOS}

Esta investigación ha sido realizada gracias a un proyecto de investigación financiado por la 


\section{EL PAPEL de LA AUTOESTIMA EN LA PREVENCIÓN DEL VIH DE JÓVENES ESPAÑOLES}

Universitat Jaume I - Fundació Bancaixa (P1 1B2006-19) y por la Fundación para la Investigación y la Prevención del Sida en España (exp. 36639/07).

\section{REFERENCIAS BIBLIOGRÁFICAS}

Ballester, R., Gil, M. D. y Giménez, C. (2007, Junio). El Cuestionario de Prevención del Sida (CPS): Análisis de la fiabilidad y validez (pp. 135). Investigación presentada en el X Congreso Nacional sobre el Sida de la Sociedad Española Interdisciplinaria de Sida, San Sebastián, España.

Crosby, R., Yarber, W. L., Sanders, S. A. y Graham, C. A. (2005). Condom discomfort and associated problems with their use among university students. Journal of American College Health, 54(3), 143-147.

Enejoh, V., Pharr, J., Mavegam, B. O., Olutola, A., Karick, H. y Ezeanolue, E. E. (2016). Impact of self esteem on risky sexual behaviors among Nigerian adolescents. Aids Care, 28(5), 672-676.

Ethier, K. A., Kershaw, T. S., Lewis, J. B., Milan, S., Niccolai, L. M. y Ickovics J. R. (2006). Self-esteem, emotional distress and sexual behavior among adolescent females: inter-relationships and temporal effects. Journal of Adolescent Health, 38(3), 268-274.

Jahoda, M. (1958). Current Concepts of Positive Mental Health. Nueva York: Basil Books.

Klein, H., Elifson, K.W. y Sterk, C.E. (2010). Self-esteem and HIV risk practices among young adult ecstasy users. Journal of Psychoactive Drugs, 42(4), 447-456.

MacDonald, T. K. y Martineau, A. M. (2002). Self-esteem, mood, and intentions to use condoms: When does low self-esteem lead to risky health behaviors? Journal of Experimental Social Psychology, 38(3), 299-306.

Martín-Albo, J., Núñez, J. L., Navarro, J. G. y Grijalvo, F. (2007). The Rosenberg Self-Esteem Scale: Translation and validation in university students. The Spanish Journal of Psychology, 10(2), 458-467.

Mruk, C. J. (2013). Self-esteem and positive psychology: Research, theory, and practice. New York: Springer Publishing Company.

Ortega, P., Mínguez, R. y Rodes, M. L. (2000). Autoestima: un nuevo concepto y su medida. Teoría de la Educación. Revista Interuniversitaria, 12, 45-66.

Orth, U., Robins, R. W. y Widaman, K. F. (2012). Life-span development of self-esteem and its effects on important life outcomes. Journal of Personality and Social Psychology, 102(6), 1271-1288.

Oxley, G. M. (2001). HIV/AIDS knowledge and self-esteem among adolescents. Clinical Nursing Research, 10(2), 214-224.

Ramiro, M. T., Teva, I., Bermúdez, M. P. y Buela-Casal, G. (2013). Social support, self-esteem and depression: Relationship with risk for sexually transmitted infections/HIV transmission. International Journal of Clinical and Health Psychology, 13, 181-188.

Rosenberg, M. (1989). Society and the adolescent self-image. Middletown, CT: Wesleyan University Press.

Salazar, L. F., Crosby, R. A., DiClemente, R. J., Wingood, G. M., Lescano, C. M., Brown, L. K. et al. (2005). Self-esteem and theoretical mediators of safer sex among African American female adolescents: implications for sexual risk reduction interventions. Health Education \& Behavior, 32(3), 413-427.

Sylvester, 0. A. (2014). Influence of self-esteem, parenting style and parental monitoring on sexual risk behaviour of adolescents in Ibadan. Gender \& Behaviour, 12(2), 6341-6353.

Træen, B., Hald, G.M., Noor, S.W., Iantaffi, A., Grey, J. y Rosser, B.R. (2014). The relationship between use of sexually explicit media and sexual risk behavior in men who have sex with men: exploring the mediating effects of sexual self-esteem and condom use self-efficacy. International Journal of Sexual Health, 26(1), 13-24. 
Ugoji, F. N. (2014). Determinants of risky sexual behaviours among secondary school students in Delta State Nigeria. International Journal of Adolescence and Youth, 19(3), 408-418.

Villegas, N., Cianelli, R., Gonzalez-Guarda, R., Kaelber, L., Ferrer, L., Peragallo, N. (2013). Predictors of self-efficacy for HIV prevention among Hispanic women in South Florida. The Journal of the Association of Nurses in AIDS Care: JANAC. 24(1), 27-37.

Yang, J., Dedovic, K. y Zhang, Q. (2010). Self-esteem and risky decisionmaking: An ERP study. NEUROCASE, 16, 512-519. 
